\title{
Dry Cupping, Ischemic Compression, or Their Combination for the Treatment of Trigger Points: A Pilot Randomized Trial
}

\author{
Mohammad Nasb, $\mathrm{PhD}_{1}^{1, *}$ Xu Qun, $\mathrm{PhD}_{1}^{2, *}$ Charith Ruckmal Withanage, MSC, \\ Xie Lingfeng, $\mathrm{PhD}^{1}$, and Chen Hong, $\mathrm{PhD}^{1}$
}

\begin{abstract}
Background: Chronic neck pain (NP) attributed to myofascial pain syndrome is one of the particularly common skeletal muscle disorder associated with the hyperirritable zone in the taut band of muscle. Trigger points (TPs) are the physical interpretation of the myofascial pain syndrome. In the United States, 30\%-85\% of pain patients have been affected by myofascial TPs.

Objectives: To reveal preliminary evidence on the clinical efficacy of ischemic compression therapy, dry cupping, and their combination on improving the TPs' pressure pain threshold (PPT), neck range of motion (NROM), and neck disability index (NDI) in patients with TPs and nonspecific NP. Besides, assess the feasibility of conducting a randomized clinical trial (RCT).

Design: A randomized pilot study was conducted on 24 patients with TPs and nonspecific NP. Patients were randomly assigned to three groups: the cupping group, the ischemic compression group, and the combination therapy group. PPT, NROM, and NDI were assessed before and after 4 weeks of treatment.

Results: The results showed a statistically significant improvement in NDI, PPT, and NROM compared with values before the treatment $(p<0.05)$ in all groups. Although no significant difference was detected between ischemic compression (IC) and dry cupping, the combination approach showed significantly higher and faster improvement $(p<0.05)$.

Conclusions: It is feasible to conduct a main RCT. Both IC and dry cupping may hold promise in treating TPs; a combination of the two therapies may provide superior improving rate.
\end{abstract}

Keywords: trigger point, ischemic compression, physical therapy, rehabilitation

\section{Introduction}

$\mathbf{N}$ ONSPECIFIC NECK PAIN (NP) is typically caused by mechanical or myofascial disorders. ${ }^{1}$ Studies suggest that active trigger points (TPs) are widespread in patients who experience nonspecific NP. ${ }^{2,3} \mathrm{TP}$ is one of the most widespread long-lasting muscle disorders affecting all ages and social groups, regardless of occupation, physical build, or physical activity levels. A TP is associated with a tender spot situated in a taut band of muscle. When this spot is manipulated, impulsive or exertion pain may be experienced. ${ }^{4}$ TPs are classified as latent or active. However, both types can lead to a decrease in functional capacity. ${ }^{5}$

TP management has become a popular topic in the literature. ${ }^{6,7}$ Several studies have attempted to examine TP complications, which have resulted in the development of various treatments. Massage, dry needle, cupping, and

\footnotetext{
${ }^{1}$ Department of Rehabilitation Medicine, Tongji Hospital, Huazhong University of Science and Technology, Wuhan, P.R. China.

${ }^{2}$ Department of Rehabilitation Medicine, Zhongnan Hospital, Wuhan University School of Medicine, Wuhan, P.R. China. *Co-first authors.

(C) Mohammad Nasb, et al. 2019; Published by Mary Ann Liebert, Inc. This Open Access article is distributed under the terms of the Creative Commons Attribution Noncommercial License (http://creativecommons.org/licenses/by-nc/4.0/) which permits any noncommercial use, distribution, and reproduction in any medium, provided the original author(s) and the source are cited.
} 
ischemic compression (IC) are the most common conventional treatment approaches for treating TP. ${ }^{8-10}$ Among these methods, IC and dry cupping show the most promise. ${ }^{11,12}$ However, comparing these methods, separately and in combination, has not been critically evaluated or compared.

IC, a manual technique that uses direct vertical pressure to the TP, is a well-established treatment method used to resolve the TP-related pain syndrome. This directed pressure is managed according to the therapist's perception of the tissue resistance of the treated soft tissue or according to the pain perceived by the patient based on a pain scale. ${ }^{13,14}$ IC is a safe, noninvasive, effective, and inexpensive way to treat muscle pain syndrome. Various types of instruments, made of wood, plastic, or rubber, are used to administer the therapy without injuring the therapist's hands.

Cupping therapy (CT), a traditional Chinese medicine therapy, has been used for $>2000$ years and uses a negative pressure mechanism. ${ }^{15}$ There are two types of cupping: wet cupping, which involves bloodletting and dry cupping, which uses only a negative pressure treatment. Its principal mechanism relies on stimulating the acupuncture points. CT is an effective way to manipulate soft tissues, however, to the best of our knowledge, the efficacy of CT on TPs had not been reported yet. ${ }^{16}$

CT and IC have been shown to decrease the pain and improve the quality of life substantially. Although few studies have evaluated the efficacy of IC on TPs, ${ }^{13,14}$ the comparison of $\mathrm{CT}$ and IC or their combination studies are lacking. This study utilizes a pretest-posttest to assess changes in pain to examine the efficacy of the treatments and prepare for a full-scale trial.

\section{Methodology}

This pilot study used a randomized controlled design. It was designed according to the Consolidated Standards of Reporting Trials Statement (CONSORT). The study was conducted in the Rehabilitation and Physical Therapy Department of Tongji Hospital, Huazhong University of Science and Technology (Wuhan, China) between February 2018 and January 2019 according to the relevant guidelines and regulations and approved by a Tongji Hospital Licensing Committee. Moreover, an authorization for the study was obtained by the Hospital Ethics Committee (approval no.: TJ-20171102) and prospectively registered with the Chinese Clinical Trials Registry (Identifier: ChiCTR800014723, date of registration January 31, 2018).

\section{Selection of participants}

Patients with chronic nonspecific NP were recruited through the e-mail system and poster advertisements in the Huazhong University of Science and Technology. Later, patients were screened using the listed TPs' diagnosing criteria, patient's History Sheet, and inclusion and exclusion criteria.

Participants were required to undergo 8 sessions for 4 weeks. Participants were informed not to use any other treatments or changing their daily life routine. Verbal and written consents were collected before the study, and participants were randomly allocated into three groups, according to the order of their participation: the cupping therapy group (CTG), the ischemic compression group (ICG), and the combination therapy group (CG). This procedure is itemized in the flow chart presented in Figure 1.

\section{Randomization and blinding}

A total of 24 patients were randomly allocated at a $1: 1: 1$ ratio to obtain cupping or IC or to get both treatments using a computerized randomization schedule. The responsible person for generating the randomization assignment was independent.

Due the nature of the interventions, the study could not be double blinded, however, a well-trained team of one evaluator, two data collectors, and two statisticians were blinded to the groups/treatment allocation. Likewise, the outcomes were documented independently and compared for agreement between data collectors. The data collectors had previous research experience, and they were trained in the Tongji Hospital Research Center.

\section{Inclusion and exclusion criteria}

Males and females 17-50 years of age with nonspecific NP for a period of $>3$ months and presented with at least one active TP at the upper trapezius muscle, with pressure pain intensity of minimum $30 \%$ (30 $\mathrm{mm}$ on the $100-\mathrm{mm}$ visual analog scale) lasting for 3 months at least, and had not used any medications or physiotherapy to alleviate pain were included in this study.

On the other hand, participants who met these criteria had been excluded:

1. NP due to trauma, whiplash, cervical disc prolapse, inflammation, malignant disease, or any other neurological and orthopedic conditions, which will affect the assessment.

2. Congenital malformation of the spine as it will alter the normal pattern of movements.

3. Had any invasive therapies in the past month.

4. Patients with sensory impairments, such as diabetic sensory neuropathy.

5. Mental disorders.

\section{TPs diagnosis}

Patients who suffered from NP for $>3$ months were examined for upper trapezius muscle TPs. The most painful TP of the upper trapezius muscle was identified and indicated with a marker pen. When bilateral NP was presented, the most painful TPs from both upper trapezius muscles were selected. TP diagnosis criteria are as follows:

1. Taut band within the muscle.

2. Exquisite tenderness at a point on the taut band.

3. Reproduction of the patient's pain.

4. Local twitch response.

5. Referred pain.

Later the diagnosis is confirmed by ultrasound imaging. ${ }^{17,18}$

\section{Outcomes}

All the measurements and therapies were conducted in erect sitting posture by the same blinded examiner for all patients at the baseline, 2 , and 4 weeks of treatment.

Pressure pain tolerance. The pressure pain tolerance (PPT) is the minimum amount of pressure that leads to discomfort or pain sensation. PPT was measured using 


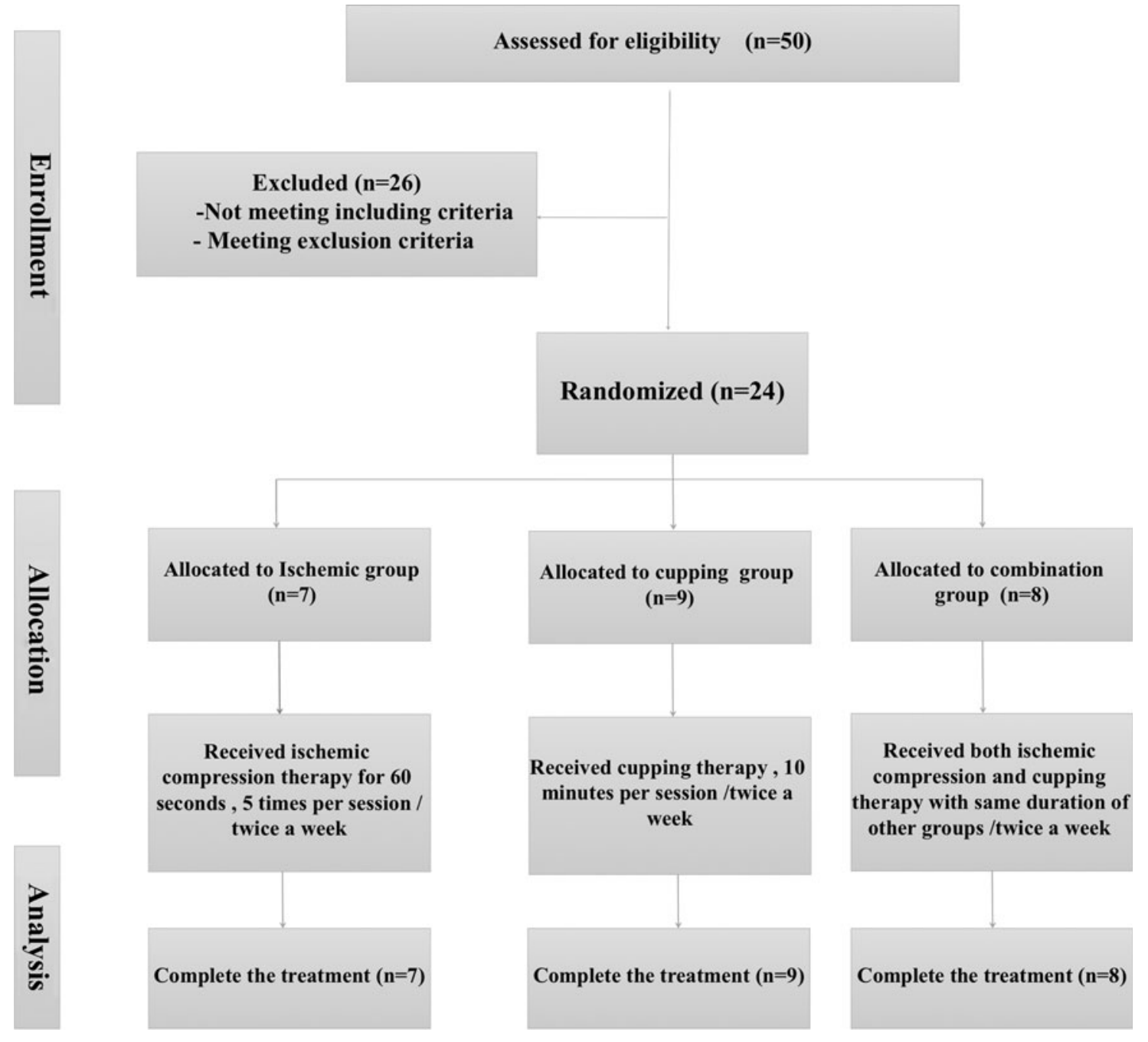

FIG. 1. Study flow chart.

(Wagner Force Dial FDK 20"; Wagner Instruments, Greenwich, CT) Algometer. ${ }^{19}$ Algometric measurements have been shown as a reliable method to assess PPT. ${ }^{20}$ It consisted of a $1 \mathrm{~cm}^{2}$ rubber tip and a dial that could display the pressure of up to $10 \mathrm{~kg}$ with graduation with $100 \mathrm{~g}$. Algometer was placed perpendicular on the marked $\mathrm{TP}$, and then the pressure was applied almost at a rate of $1 \mathrm{~kg} /\left(\mathrm{cm}^{2} \cdot \mathrm{sec}\right)$ until the pressure caused a pain. The maximum pressure displayed by the Algometer was recorded. Three measurements were obtained, and the mean value was calculated.

Neck disability index. Neck disability index (NDI) is a self-assessed evaluation tool to measure disability; it is commonly hired to assess the treatment effects on neck disorders. NDI has been involved in lots of studies according to a recent search on PubMed. Moreover, it has been broadly examined for repeatability and validity. ${ }^{21,22}$

NDI has 10 questions, and the total score is calculated out of 50. The final outcome score is interpreted as following; 0-4=none; 5-14=mild; $15-24=$ moderate; $25-34=$ severe; over $34=$ complete. The minimal clinically important change for nonspecific NP patients range from 3.5 to 7.5 out of $50 .^{23}$
Neck range of motion. Neck range of motion (NROM) was measured using the GLOBAL POSTURAL SYSTEM (GPS) device (GPS 600; Chinesport SpA, Udine, Italy) and the GPS 5.0 software. This system uses a digital cervical test helmet with GPS software to measure the NROM in frontal, sagittal, and transverse planes; it could measure lateral flexion, rotation extension and flexion. Before using the helmet, it has to be calibrated by moving in the three individual planes. Then the helmet was positioned on the patient's head and was instructed to move their head as instructed. GPS software shows the angle of the movements. ${ }^{24}$

\section{Interventions}

Cupping therapy. The participants received CT for 10 min on the marked TP using a $12^{\prime \prime}$ plastic set with a hand vacuum pump (OMAIASHIBAGUANQ International Supplies Co., Ltd., China). The vacuum cup size was determined according to the area on which the TP was situated (A02 and A03 cups were used). The skin and the cup were disinfected with alcohol, and the cup was placed on the marked TP, followed by a tolerable intensity of pumping. The cup was then kept on the TP for $10 \mathrm{~min}$. When treating bilateral TPs, one cup was placed on the TP on the right side, 
Table 1. Demographic Data

\begin{tabular}{lccr}
\hline Characteristics & $\begin{array}{c}\text { Cupping } \\
\text { therapy }\end{array}$ & $\begin{array}{c}\text { Ischemic compression } \\
\text { therapy }\end{array}$ & $\begin{array}{c}\text { Combination } \\
\text { therapy }\end{array}$ \\
\hline Number of patients & 9 & 7 & 8 \\
Age (years), mean (SD) & $31(12.9)$ & $33.1(9.3)$ & $29(11.5)$ \\
Gender, $n$ (\%) of female & $6(66.6)$ & $4(57.1)$ & $5(62.5)$ \\
Duration of neck pain episode, (months) & $33.2(16.2)$ & $29.6(15.7)$ & $37.5(13.9)$ \\
VAS score, mean (SD) & $59.7(24.4)$ & $61.7(28.7)$ & $62.9(21.5)$ \\
Number of total TPs & 13 & 9 & 11 \\
\hline
\end{tabular}

SD, standard deviation; TPs, trigger points; VAS, visual analog scale.

and another was placed on the left side with a minimum delay. The time was measured after affixing the plastic vacuum on the left side of the TP. ${ }^{16,25,26}$

Ischemic compression. According to a latest systematic review, IC is a frequently hired method in the pain management of TP patients. ${ }^{13}$ This approach has been indicated to decrease pain intensity and increase range of motion (ROM) of neck flexion and extension in patients with TPs in the upper trapezius muscle.

The therapist applied five continuous deep pressure compressions for 1 min on the marked TPs, with a 1-min rest between every compression. Pressure released when the TP tension decreased, pain relieved or the treatment passed 1 min, whichever came first. ${ }^{2}$

Combination therapy. CG received both IC and CT, respectively. First IC was administered to the selected TP then followed by CT with minimum delay. It took $\sim 20 \mathrm{~min}$ to treat one TP.

\section{Statistical analyses}

Data were recorded in the case report form. The most painful TP was selected when bilateral TPs were presented. The values of all groups are demonstrated as the mean \pm standard deviation. All data were analyzed using SPSS version 22.0 statistical software.

After checking the normal distribution of the continuous variables, parametric statistical tests were used. Analysis of variance test was performed to calculate the variance between the three groups, whereas paired $t$-test was performed to calculate the statistical difference within groups.

A sample size of 24 patients was considered appropriate for this preliminary study, as this study aimed to examine the feasibility of randomized clinical trial (RCT). On the other hand, the effect size calculation will help in running appropriate power analyses in future full-scale RCT. We found the overall effect sizes $(f=0.5$, and $f=0.6)$ for the NDI and PPT, respectively, were considered a "medium" effect size. As the effect size of PPT $(f=0.6)$ was higher, it is suggested to be the primary outcome in the future RCT.

\section{Results}

\section{Demographic data and clinical features}

A total number of 24 patients had participated in the study. Average NP duration was 33.4 months. All patients had completed the treatment. The demographic data of the three groups are mentioned in Table 1 . There was no noteworthy variance among the three groups at baseline measures in demographic data and clinical features.

\section{Improvements within groups}

Cupping therapy group. Comparison of the results at baseline and after 4 weeks of CT are presented in Table 2, there is a significant improvement in the PPT score at 4 weeks of treatment $(p<0.01)$. When compared with the baseline score, NDI showed significant decreasing after 2 weeks of treatment $(p<0.01)$. The NROM values in CTG also were significantly improved at 4 weeks of treatment, except for the side rotation ROM $(p<0.05)$.

Ischemic compression therapy group. Comparison of the outcomes before and after the ischemic compression therapy (ICT) are presented in Table 2. A significant increase in the PPT score was achieved at 4 weeks of treatment $(p<0.05)$. Contrarily, no significant changes in NDI were noticed after 2 weeks of treatment, whereas the

Table 2. Comparison of the Outcome Measures Between Baseline and Post-treatment in All Groups by Mean (Standard Deviation)

\begin{tabular}{|c|c|c|c|c|c|c|c|}
\hline Scale & Phase & $\begin{array}{l}\text { Cupping } \\
\text { therapy }\end{array}$ & $\begin{array}{c}\text { Ischemic } \\
\text { compression } \\
\text { therapy }\end{array}$ & $\begin{array}{c}\text { Combination } \\
\text { therapy }\end{array}$ & $\begin{array}{c}\text { Cupping vs ischemic } \\
\text { compression, } \\
\text { p-value }\end{array}$ & $\begin{array}{l}\text { Cupping vs } \\
\text { combination, } \\
\text { p-value }\end{array}$ & $\begin{array}{c}\text { Ischemic } \\
\text { compression vs } \\
\text { combination, p-value }\end{array}$ \\
\hline \multirow[t]{2}{*}{ NDI } & Baseline & $10.56(3.3)$ & $12.71(4.3)$ & $10.62(3.9)$ & & \multirow[t]{2}{*}{0.04} & \multirow[t]{2}{*}{0.02} \\
\hline & Post-treatment & $3.70(1.2)$ & $5.50(2.1)$ & $2.90(2.1)$ & 0.42 & & \\
\hline \multirow[t]{2}{*}{ PPT } & Baseline & $4.48(1.4)$ & $5.71(1.5)$ & $5.62(1.6)$ & & \multirow[t]{2}{*}{0.03} & \multirow[t]{2}{*}{0.01} \\
\hline & Post-treatment & $5.62(1.6)$ & 6.28 (1.6) & 7.93 (1.4) & 0.25 & & \\
\hline
\end{tabular}

NDI, neck disability index; PPT, pressure pain threshold. 
significant decreasing was noticed at 4 weeks of treatment $(p<0.05)$. The NROM values in ICG also showed significant increasing in all aspects other than left side rotation at 4 weeks of treatment with $p<0.05$ (Table 3 ).

Combined therapy group. Presentations of the results (pre and post-treatment) for the combination therapy are exhibited in Table 2. A noteworthy rising in the PPT score was accomplished at the 2 weeks of treatment $(p<0.05)$, combined therapy group showed the greatest increase in PPT mean $\left(1.638 \mathrm{~kg} / \mathrm{cm}^{2}\right)$ between the first and eighth sessions.

Moreover, a significant declining was observed in NDI at 2 weeks of treatment $(p<0.01)$. Likewise, the NROM values in the combination group showed significant improvement in all aspects at 4 weeks of treatment with $p<0.05$ (Table 3).

\section{Comparison of the three groups}

Although all groups exhibited a relatively high substantial improvement regarding PPT, NDI, and neck NROM, There were no statistically significant differences in treatment outcome measures between ICT and CT groups at 4 weeks of treatment $(p>0.05)$.

On the other hand, the combination therapy showed a statistically significant decrease in the score of NDI and increase in the PPT (Table 2). Although, the combination group showed an increase in all aspects of NROM, no significant difference was detected among the three approaches regarding increasing the NROM.

\section{Discussion}

The present pilot study has confirmed the feasibility to conduct a full-scale trial. We have shown that the combination of ICT and CT presented more promising than using each approach individually in treating patients with upper trapezius myofascial TPs.

One encouraging part of our study was that we compared well-known treatment methods for TPs. To the best of our knowledge, this is the first research study to clinically examine the effects of CT and the combination of compression and $\mathrm{CT}$ on releasing myofascial TPs. We mainly focused on the PPT, NDI, and NROM; improving PPT and NDI are a vital indicator of improved sensitivity and treatments directed at TPs that lead to improved clinical outcomes, such as reduced pain. ${ }^{12,27}$

IC is a popular and effective manual therapy method for treating TP-related pain. ${ }^{14}$ It has shown short-term and longterm positive effects on increasing PPT, joint ROM, and muscle strength. ${ }^{28,29}$ It is a well-researched and standardized method of therapy to treat TPs due to its ability to increase local blood circulation. In the present study, it is clear that ICT could improve the PPT of the TP. ${ }^{30}$

Moreover, several studies presented the short-term effect of ICT to diminish NDI scores within 2 weeks of treatment. On the other hand, the long-term effect was not sufficient to improve the NDI score for the 6 months' therapeutic course. ${ }^{12}$ Likewise, in the current study, the NDI score showed decreasing along the treatment.

CT has been used to treat pain symptoms for thousands of years to the best of our knowledge; however, there was no literature on specifically treating TPs. Cupping was generally

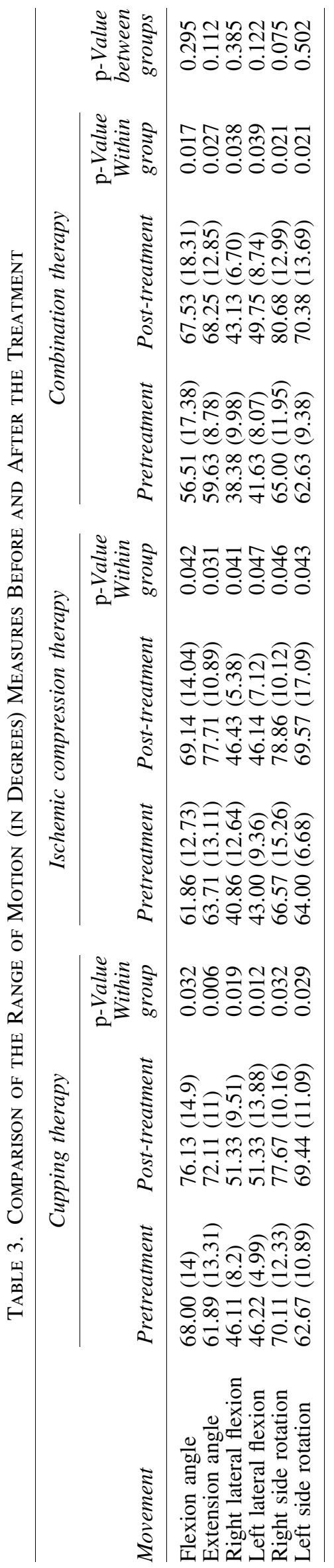


used to stimulate acupuncture points. Some recent studies had revealed the capability of cupping to increase the blood circulation at the cupping point. ${ }^{31}$ It also showed some influence on increasing the parasympathetic activity, which could also increase the peripheral blood supply and reduction in pain. ${ }^{32,33}$

Although there are no earlier studies using CT to treat TPs, CT has been shown to increase PPT in patients with nonspecific NP. ${ }^{33}$ This study found a statistically significant improvement $(p=0.0242)$ in PPT following 4 weeks of treatment.

In the same vein, ICT improved NDI scores at more than a single CT session on nonspecific NP patients. ${ }^{34}$ In this study, all patients experienced a statistically significant improvement in NDI scores within the duration of the therapeutic course.

A group receiving a combination of CT and ICT showed greater improvement in PPT $(p<0.01)$ with an average of $1.63 \mathrm{~kg} / \mathrm{cm}^{2}$; the CT-only group showed less improvement, with an average of $1.133 \mathrm{~kg} / \mathrm{cm}^{2}$. Although both individual treatments were effective in reducing NDI, the combination approach was abundantly superior. As hypothesized, TP generation and persistence was closely related to the hypoxic environment. Consequently, the combination of the two therapies had a superior outcome to TP therapy. The CG also achieved significant improvement more quickly than the groups using individual approaches, with a significant improvement represented by $p<0.05$ on the PPT scale after 2 weeks of treatment. The individual approaches took the full course duration to significantly increase PPT, whereas the combination group accomplished it in 2 weeks of treatment.

ROM improvement, lateral flexion, and extension and flexion are the most clinically significant movements for the upper trapezius muscle. Several studies that measured the effects of ICT on cervical lateral flexion revealed that one or more sessions could improve the lateral flexion of the neck. ${ }^{35}$ However, no study had evaluated the effects of cupping on NROM in patients with TPs. In this study, flexion angle, extension angle, and lateral flexion angle improved in all groups. Although CT and ICT groups showed statistically significant improvement in some movement, no significant difference between the three therapy groups was detected. However, the combination therapy was significantly more efficient than individual treatments for all the items assessed, excluding NROM.

The study outcomes will assist in planning the next phase of the research. The results suggested the importance of testing the treatment methods on a larger scale besides confirming the results of this preliminary study in a more representative sample.

We learned that the combination group achieved better improvement than other treatment groups regarding NDI and PPT. These results suggested that future research should use a bigger sample size and examine the long-term effects of three treatments, which may also show the best intervention for improving NROM. However, the study had the following limitations. We did not identify the optimum duration of cupping or ICs and the potential placebo effect should be studied. Thus, further research is recommended highly to confirm our findings.

In conclusion, although CT and IC showed potential in treating TPs, it is suggested that the combination of CT and
IC is a more promising treatment regime for TPs than either therapy alone, and further evidence through a high-quality RCT is highly recommended.

\section{Author Disclosure Statement}

No competing financial interests exist.

\section{Funding Information}

The authors have no funding, financial relationships, or conflicts of interest to disclose.

\section{References}

1. McLean SM, May S, Klaber-Moffett J, et al. Risk factors for the onset of non-specific neck pain: A systematic review. J Epidemiol Community Health 2010;64:565-572.

2. Simons DG, Travell JG, Simons LS. Travell \& Simons' Myofascial Pain and Dysfunction: Upper Half of Body (Vol. 1). Philadelphia, PA: Lippincott Williams \& Wilkins, 1999.

3. Fernández-de-Las-Peñas C, Alonso-Blanco C, Miangolarra JC. Myofascial trigger points in subjects presenting with mechanical neck pain: A blinded, controlled study. Manual Ther 2007;12:29-33.

4. Gerwin RD. Diagnosis of myofascial pain syndrome. Phys Med Rehabil Clin N Am 2014;25:341-355.

5. Alvarez DJ, Rockwell PG. Trigger points: Diagnosis and management. Am Fam Physician 2002;65:653-662.

6. Kisilewicz A, Janusiak M, Szafraniec R, et al. Changes in muscle stiffness of the Trapezius muscle after application of ischemic compression into myofascial trigger points in professional basketball players. J Hum Kinet 2018;64:35-45.

7. Choi YH, Jung SJ, Lee CH, Lee SU. Additional effects of transcranial direct-current stimulation and trigger-point injection for treatment of myofascial pain syndrome: A pilot study with randomized, single-blinded trial. J Altern Complement Med 2014;20:698-704.

8. Moraska AF, Schmiege SJ, Mann JD, et al. Responsiveness of myofascial trigger points to single and multiple trigger point release massages: A randomized, placebo controlled trial. Am J Phys Med Rehabil 2017;96:639-645.

9. Tang L, Li Y, Huang Q-M, Yang Y. Dry needling at myofascial trigger points mitigates chronic post-stroke shoulder spasticity. Neural Regen Res 2018;13:673-673.

10. Behrangrad S, Kamali F. Comparison of ischemic compression and lumbopelvic manipulation as trigger point therapy for patellofemoral pain syndrome in young adults: A double-blind randomized clinical trial. J Bodyw Mov Ther 2017;21:554-564.

11. Cao H, Li X, Liu J. An updated review of the efficacy of cupping therapy. PLoS One 2012;7:e31793.

12. Cagnie B, Dewitte V, Coppieters I, et al. Effect of ischemic compression on trigger points in the neck and shoulder muscles in office workers: A cohort study. J Manipulative Physiol Ther 2013;36:482-489.

13. Cagnie B, Castelein B, Pollie F, et al. Evidence for the use of ischemic compression and dry needling in the management of trigger points of the upper trapezius in patients with neck pain: A systematic review. Am J Phys Med Rehabil 2015;94:573-583.

14. Bialosky JE, Bishop MD, Price DD, et al. The mechanisms of manual therapy in the treatment of musculoskeletal pain: A comprehensive model. Manual Ther 2009;14:531-538. 
15. Lone AH, Ahmad T, Anwar M, et al. Leech therapy-a holistic approach of treatment in unani (greeko-arab) medicine. Anc Sci Life 2011;31:31.

16. Bridgett R, Klose P, Duffield R, et al. Effects of cupping therapy in amateur and professional athletes: Systematic review of randomized controlled trials. J Altern Complement Med 2018;24:208-219.

17. Simons DG. Diagnostic criteria of myofascial pain caused by trigger points. J Musculoskelet Pain 1999;7:111-120.

18. Kumbhare DA, Elzibak AH, Noseworthy MD. Assessment of myofascial trigger points using ultrasound. Am J Phys Med Rehabil 2016;95:72-80.

19. Mikkelsson M, Latikka P, Kautiainen H, et al. Muscle and bone pressure pain threshold and pain tolerance in fibromyalgia patients and controls. Arch Phys Med Rehabil 1992;73:814-818.

20. Park G, Kim CW, Park SB, et al. Reliability and usefulness of the pressure pain threshold measurement in patients with myofascial pain. Ann Rehabil Med 2011;35:412-417.

21. Vernon H. The neck disability index: State-of-theart, 1991-2008. J Manipulative Physiol Ther 2008;31: 491-502.

22. Young IA, Cleland JA, Michener LA, et al. Reliability, construct validity, and responsiveness of the neck disability index, patient-specific functional scale, and numeric pain rating scale in patients with cervical radiculopathy. Am J Phys Med Rehabil 2010;89:831-839.

23. Young BA, Walker MJ, Strunce JB, et al. Responsiveness of the neck disability index in patients with mechanical neck disorders. Spine J 2009;9:802-808.

24. Toprak M, Alptekin HK, Turhan D. Correction to: P-12 assessment of symmetrigraph and global postural system results for the posture analysis of the healthy individuals. Chiropr Man Therap 2018;26:20-20.

25. Cramer H, Lauche R, Hohmann C, et al. Randomized controlled trial of pulsating cupping (pneumatic pulsation therapy) for chronic neck pain. Complement Med Res 2011;18:327-334

26. Chi LM, Lin LM, Chen CL, et al. The effectiveness of cupping therapy on relieving chronic neck and shoulder pain: A randomized controlled trial. Evid Based Complement Alternat Med 2016;2016:1-7.

27. Gemmell H, Miller P, Nordstrom H. Immediate effect of ischaemic compression and trigger point pressure release on neck pain and upper trapezius trigger points: A randomised controlled trial. Clin Chiropr 2008;11:30-36.

28. Wilke J, Vogt L, Banzer W. Immediate effects of selfmyofascial release on latent trigger point sensitivity: A randomized, placebo-controlled trial. Biol Sport 2018;35: 349-354.
29. Aguilera FJM, Martín DP, Masanet RA, et al. Immediate effect of ultrasound and ischemic compression techniques for the treatment of trapezius latent myofascial trigger points in healthy subjects: A randomized controlled study. J Manipulative Physiol Ther 2009;32:515-520.

30. Hou CR, Tsai LC, Cheng KF, et al. Immediate effects of various physical therapeutic modalities on cervical myofascial pain and trigger-point sensitivity. Arch Phys Med Rehabil 2002;83:1406-1414.

31. Hong SH, Wu F, Lu X, et al. Study on the mechanisms of cupping therapy. Zhongguo Zhen Jiu 2011;31:932-934.

32. Lauche R, Cramer H, Hohmann $\mathrm{C}$, et al. The effect of traditional cupping on pain and mechanical thresholds in patients with chronic nonspecific neck pain: A randomised controlled pilot study. Evid Based Complement Alternat Med 2012;2012:1-10.

33. Emerich M, Braeunig M, Clement HW, et al. Mode of action of cupping-Local metabolism and pain thresholds in neck pain patients and healthy subjects. Complement Ther Med 2014;22:148-158.

34. Kim KH, Kim TH, Hwangbo M, Yang GY. Anaemia and skin pigmentation after excessive cupping therapy by an unqualified therapist in Korea: A case report. Acupunct Med 2012;30:227-228.

35. Ganesh GS, Singh H, Mushtaq S, et al. Effect of cervical mobilization and ischemic compression therapy on contralateral cervical side flexion and pressure pain threshold in latent upper trapezius trigger points. J Bodyw Mov Ther 2016;20:477-483.

Address correspondence to: Chen Hong, PhD

Department of Rehabilitation Medicine Tongji Hospital

Huazhong University of Science and Technology

Jiefang Avenue

Qiaokou Qu, Wuhan 430030

P.R. China

E-mail: chenhong1129@hotmail.com

Xie Lingfeng, PhD

Department of Rehabilitation Medicine

Tongji Hospital

Huazhong University of Science and Technology

Jiefang Avenue

Qiaokou Qu, Wuhan 430030

P.R. China

E-mail: xielingfeng1983@126.com 